\title{
Optimized Self Reconfigurable Wireless Mesh Networks
}

\author{
M. V. Jaishree ${ }^{1}$, Ms. N. Revathi ${ }^{2}$ \\ ${ }^{l}$ Dept. of Information and Communication Engineering, Sri Venkateswara College of Engineering \\ Chennai, India \\ ${ }^{2}$ Dept. of Information and Communication Engineering, Sri Venkateswara College of Engineering \\ Chennai, India
}

\begin{abstract}
Wireless Mesh Networks (WMNs) are being developed actively and deployed widely for a variety of applications, such as public safety, environment monitoring, and citywide wireless Internet services. They have also been evolving in various forms (e.g., using multiradio/channel systems to meet the increasing capacity demands by the above-mentioned and other emerging applications. However, due to heterogeneous and fluctuating wireless link conditions, preserving the required performance of such WMNs is still a challenging problem. . For example, some links of a WMN may experience significant channel interference from other coexisting wireless networks. Some parts of networks might not be able to meet increasing bandwidth demands from new mobile users and applications. The above problem in the WMNs is overcome by the proposed system i.e. autonomous network reconfiguration system (ARS) that allows a multiradio WMN(mr-WMN) to autonomously reconfigure its local network settings- channel, radio, and route assignment - for real-time recovery from link failures. This show that ARS outperforms existing failure-recovery schemes in improving channel-efficiency by more than $90 \%$ and in the ability of meeting the applications' bandwidth demands by an average of $200 \%$.This paper presents shows that ARS decouples network reconfiguration from flow assignment and routing. This is the first step to solve optimization problem in giving best effort service to network failures is proposed. In this a threshold value is set for every node is the modification is proposed. When a node want to send packet from source to destination,it verify its threshold value,whether it reach its limit or not. When it reaches it limit,it choose another node to send data.It chooses it route with minimum path cost value and minimum delay time.With this consideration,some considerable amount of packet loss will be less when compared to the ARS system.
\end{abstract}

\section{INTRODUCTION}

As various wireless networks evolve into the next generation to provide better services, a key technology, wireless mesh networks (WMNs), has emerged recently. In WMNs, nodes are comprised of mesh routers and mesh clients. Each node operates not only as a host but also as a router,forwarding packets on behalf of other nodes that may not be within direct wireless transmission range of their destinations. A WMN is dynamically self-organized and self-configured, with the nodes in the network automatically establishing and maintaining mesh connectivity among themselves(creating, in effect, an ad hoc network). This feature brings many advantages to WMNs such as low up-front cost, easy network maintenance,robustness, and reliable service coverage.

Conventional nodes (e.g., desktops, laptops,PDAs, PocketPCs, phones, etc.) equipped with wireless network interface cards (NICs) can connect directly to wireless mesh routers. Customers without wireless NICs can access WMNs by connecting to wireless mesh routers through, for example, Ethernet. Thus, WMNs will greatly help the users to be always-on-line anywhere anytime.Moreover, the gateway/bridge functionalities in mesh routers enable the integration of WMNs with various existing wireless networks such as cellular, wireless sensor, wireless-fidelity (Wi-Fi), worldwide inter-operability for microwave access (WiMAX), WiMedia networks. Consequently, through an integrated WMNs, the users of existing network can be provided with otherwise impossible services of these networks.

WMNs is a promising wireless technology for numerous applications,e.g., broadband home networking,community and neighborhood networks,enterprise networking, building automation,etc. It is gaining significant attention as a possible way for cash strapped Internet service providers (ISPs), carriers, and others to roll out robust and reliable wireless broadband service access in a way that needs minimal up-front investments. With the capability of self-organization and self configuration,WMNs can be deployed incrementally,one node at a time, as needed. As more nodes are installed, the reliability and connectivity for the users increase accordingly.

WMNs are being developed actively and deployed widely for a variety of applications, such as public safety, environment monitoring, and citywide wireless Internet services. They have also been evolving in various forms (e.g., using multiradio/channel systems to meet the increasing capacity demands by the above-mentioned and other emerging applications. However, due to heterogeneous and fluctuating wireless link conditions, preserving the required performance of such WMNs is still a challenging problem. For example, some links of a WMN may experience significant channel interference from other coexisting wireless networks. Some parts of networks might not be able to meet increasing bandwidth demands from new mobile users and applications. Links in a certain area (e.g., a hospital or police station) might not be able to use some frequency channels because of spectrum etiquette or regulation.

\section{RELATED WORK}

This paper [1] describes the Wireless Mesh Networks.A WMN is dynamically self-organized and self-configured, with the nodes in the network automatically establishing and maintaining mesh connectivity among themselves (creating, in effect, an ad hoc network). This feature brings many advantages to WMNs such as low up-front cost, easy network maintenance, robustness, and reliable service coverage. Conventional nodes (e.g., desktops, laptops, PDAs, Pocket PCs, phones, etc.) equipped with wireless network interface cards (NICs) can connect directly to wireless mesh routers. Customers without wireless NICs can access WMNs by 
connecting to wireless mesh routers through, for example, Ethernet. Thus, WMNs will greatly help the users to be always-on-line anywhere anytime. Moreover, the gateway/bridge functionalities in mesh routers enable the integration of WMNs with various existing wireless networks such as cellular, wireless sensor, wireless-fidelity (WiFi),worldwide inter-operability for microwave access (WiMAX), WiMedia networks. Consequently, through an integrated WMN, the users of existing network can be provided with otherwise impossible services of these networks. WMNs can be built up based on existing technologies. Some companies already have products for sale, while other companies have started to deploy WMNs in various application scenarios.

This paper [2] describes how the capacity of a static multichannel network scales as the number of nodes, n, increases. The total data rate is divided equally among the channels, and therefore the data rate supported by any one of the $\mathrm{c}$ channels is $\mathrm{W}=\mathrm{c}$. This was the channel model used by Gupta and Kumar, and we primarily use this model in our analysis. In this model, as the number of channels increases, each channel supports a smaller data rate. This model is applicable to the scenario where the total available bandwidth is fixed, and new channels are created by partitioning existing channels.

This paper [3] describes the capacity problem in wireless mesh networks can be alleviated by equipping the mesh routers with multiple radios tuned to non-overlapping channels. However, channel assignment presents a challenge because co-located wireless networks are likely to be tuned to the same channels. The resulting increase in interference can adversely affect performance. This paper presents an interference-aware channel assignment algorithm and protocol for multi-radio wireless mesh networks that address this interference problem. The proposed solution intelligently assigns channels to radios to minimize interference within the mesh network and between the mesh network and co-located wireless networks. It utilizes a novel interference estimation technique implemented at each mesh router.

This paper [4] have shown that when nodes are equipped with multiple heterogenous radios, it is important to select channel diverse paths in addition to accounting for the loss rate and bandwidth of individual links. We have implemented a routing protocol MR-LQSR (Multi-Radio Link-Quality Source Routing) with a new metric WCETT (Weighted Cumulative Expected Transmission Time) to accomplish this task, and compared its performance to other routing metrics in a multiradio testbed. Our results show that WCETT outperforms previously-proposed metrics.

This paper [5] describe the Joint channel assignment and routing for throughput optimization.Wireless broadband networks are being increasingly deployed in a multi-hop wireless mesh network (WMN) configuration. These WMNs are being used on the last mile for extending or enhancing Internet connectivity for mobile clients located on the edge of the wired network. Equipping wireless routers with multiple radios further improves the capacity by transmitting over multiple radios simultaneously using orthogonal channels. Efficient channel assignment and routing is essential for throughput optimization of mesh clients. we mathematically formulate the joint channel assignment and routing problem, taking into account the interference constraints, the number of channels in the network and the number of radios available at each mesh router. We then use this formulation to develop a solution for our problem that optimizes the overall network throughput subject to fairness constraints on allocation of scarce wireless capacity among mobile clients.

This paper [6] have identify such that fixed multi-channel multi-hop wireless networks with multiple radios per node as MC-MR networks. One of the main goals in the design of fixed wireless broadband networks is capacity planning. Within this realm,given a set of end-to-end demands, there are multiple design goals for which a network can be optimized, for example, maximizing a function of the rates where the function can be chosen to be an user utility function or a network price function, ensuring some notion of rate fairness, or minimizing end-to-end delays. For every design goal, one needs to be able to design routes, assign channels and schedule packets to meet these goals. Our MC-MR networks model provides both necessary and sufficient conditions for a feasible channel assignment and schedule in the network, using the protocol model of interference. Among the many possible capacity planning objectives, in this paper, we consider the fundamental problem of feasibility of a given end-to-end demand vector,and design a fast primal-dual algorithm for a fully polynomial time approximation solution (FPTAS) which provides an upper bound to the feasibility problem. Our algorithm is extensible to the optimization of similar rate-based objective functions that are useful for characterizing the capacity region.

\section{SYSTEM ANALYSIS}

WMNs are being developed actively and deployed widely for a variety of applications, such as public safety, environment monitoring, and citywide wireless Internet services . However, due to heterogeneous and fluctuating wireless link conditions, preserving the required performance of such WMNs is still a challenging problem. Even though many solutions for WMNs to recover from wireless link failures have been proposed, they still have several limitations.The following subtopics describes the existing system and the proposed system.

\subsection{EXISTING SYSTEM}

We proposed an autonomous network reconfiguration system (ARS) that allows a multiradio WMN (mr-WMN) to autonomously reconfigure its local network settingschannel, radio, and route assignment - for real-time recovery from link failures. In its core, ARS is equipped with a reconfiguration planning algorithm that identifies local configuration changes for the recovery while minimizing changes of healthy network settings. 
* ARS first searches for feasible local configuration changes available around a faulty area, based on current channel and radio associations. Then, by imposing current network settings as constraints, ARS identifies reconfiguration plans that require the minimum number of changes for the healthy network settings.

* Next, ARS also includes a monitoring protocol that enables a WMN to perform real-time failure recovery in conjunction with the planning algorithm. The accurate link-quality information from the monitoring protocol is used to identify network changes that satisfy applications' new QoS demands or that avoid propagation of QoS failures to neighboring links (or "ripple effects").

* Based on the measurement information, ARS detects link failures and/or generates QoS-aware network reconfiguration plans upon detection of a link failure. ARS has been implemented and evaluated extensively via experimentation on our multi radio WMN test-bed as well as via Java. Our evaluation results show that ARS outperforms existing failure-recovery methods, such as static or greedy channel assignments, and local rerouting. First, ARS's planning algorithm effectively identifies reconfiguration plans that maximally satisfy the applications' QoS demands, accommodating twice more flows than static assignment. Next, ARS avoids the ripple effect via QoS-aware reconfiguration planning, unlike the greedy approach. Third, ARS's local reconfiguration improves network throughput and channel efficiency by more than $26 \%$ and $92 \%$, respectively, over the local rerouting scheme.

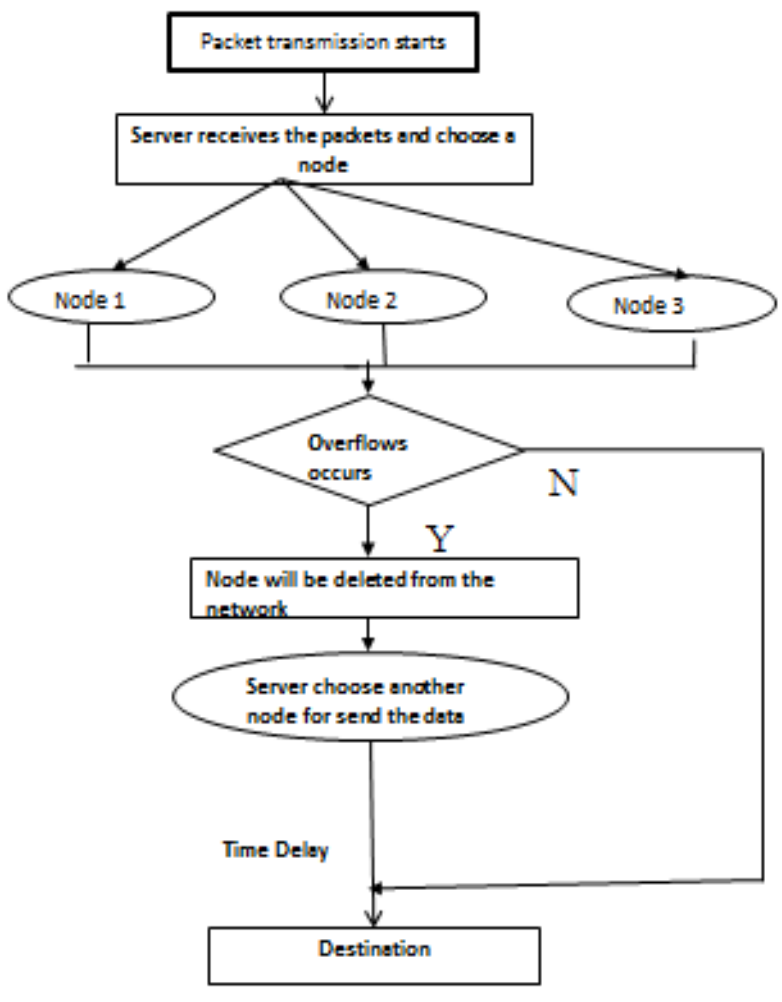

Fig. 1: EXISTING SYSTEM

\subsection{PROPOSED SYSTEM}

ARS decouples network reconfiguration from flow assignment and routing.Reconfiguration might be able to achieve better performance if two problems are jointly considered.A couple of proposals to solve this problem only provide theoretical bounds without considering practical system issues. Even though its design goal is to recover from network failures as a best-effort service.ARS is the first step to solve this optimization problem.In this, a threshold value is set for every node. When a node want to send packet from source to destination,it verify its threshold value, whether it reach its limit or not. When it reaches it limit, it choose another node to send data.It chooses it route with minimum path cost value and minimum delay time.With this consideration, some considerable amount of packet loss will be less when compared to the ARS system. 


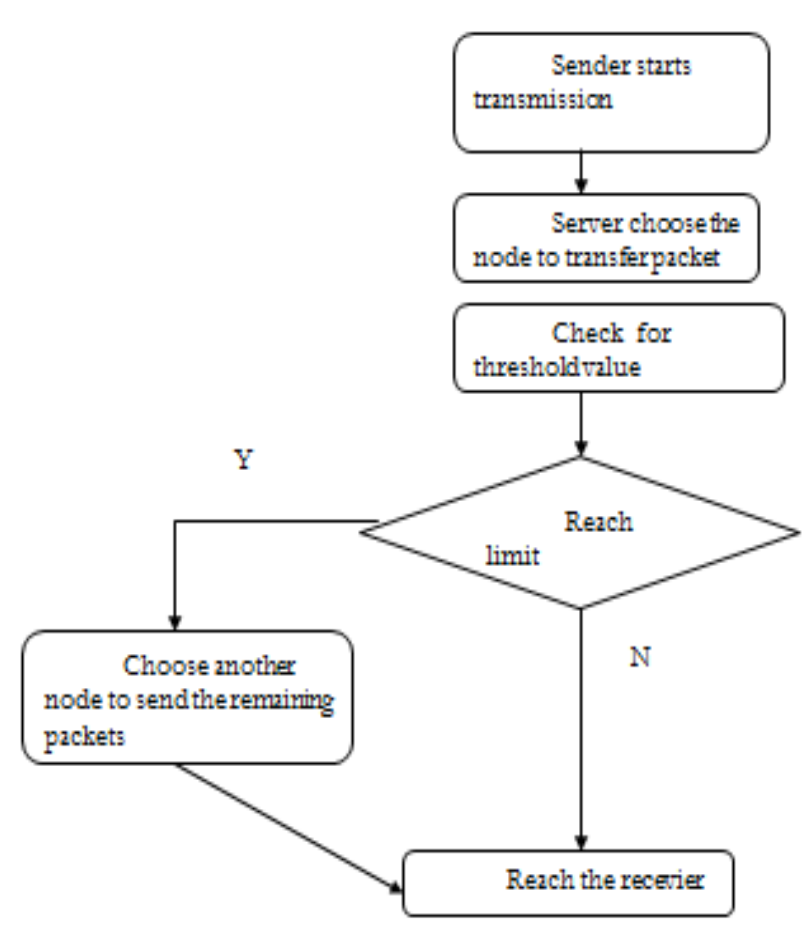

Fig.2: PROPOSED SYSTEM

\section{SYSTEM REQUIREMENTS Hardware Requirements}

- Processor IV

- RAM : $\quad 512 \mathrm{MB}$

- HDD : $\quad 80 \mathrm{~GB}$

Software Requirements

- Platform

:Windows Xp

- Front End

: Java JDK1.5,

- $\quad$ Back End :MS SQL server

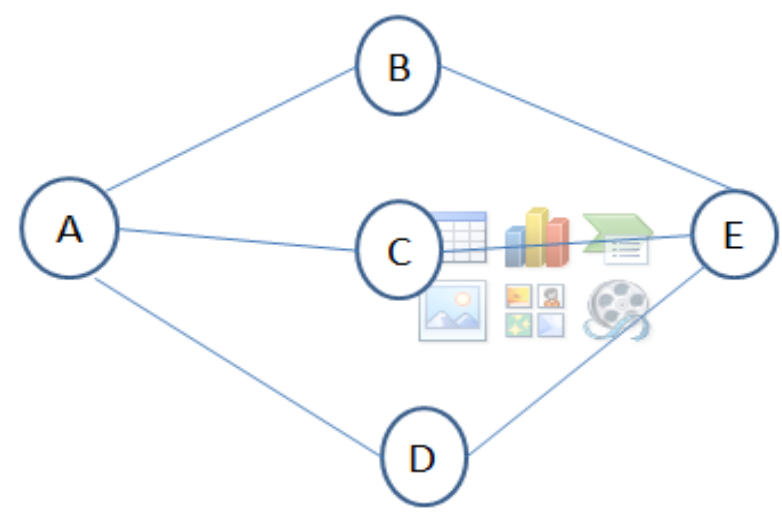

Fig.3:NETWORK MODEL

\subsection{MODULES AND ITS RESULTS}

\subsubsection{SERVER CONFIGURATION}

In this module we create user screen for creation of node. We create a network topology to send the data. Network has many no of node details. It maintains the connection details also. Nodes are interconnected and exchange data directly with each other nodes. Nodes are connecting with other nodes in the network. Network server maintains the node ip address, port details and status. Node give request to server and get the node details from server.A network are assumed to consist of mesh nodes and one control gateway. Fig 4 shows node construction and its connection details.

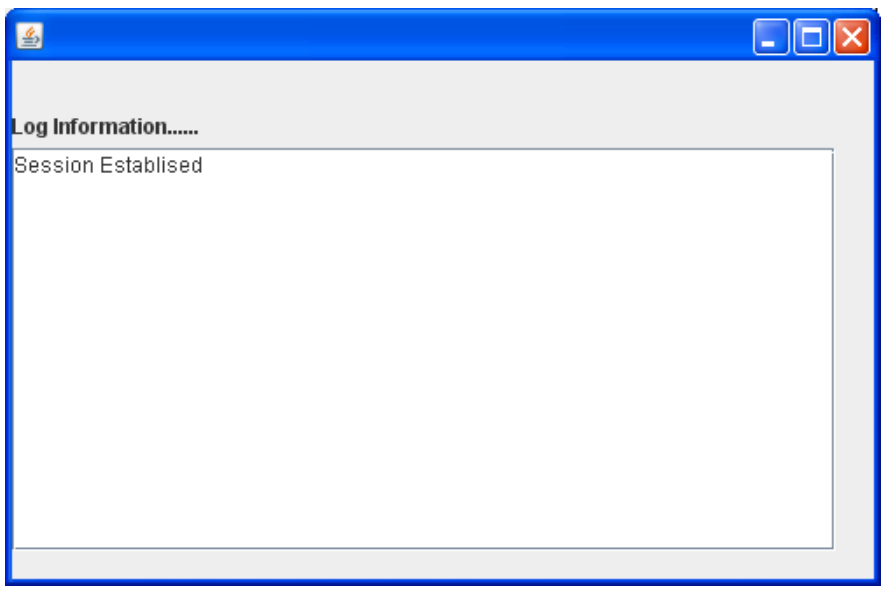

\section{SYSTEM IMPLEMENTATION}

We first introduce the network model and assumptions to be used in this paper.Next we describes the various modules of the system have been implemented. The implementation results are discussed in the following sub topics.

\subsection{NETWORK MODEL AND ASSUMPTION}

We consider below network model for our construction of nodes and consider one hop count for all the nodes and assign each node with the threshold value. 


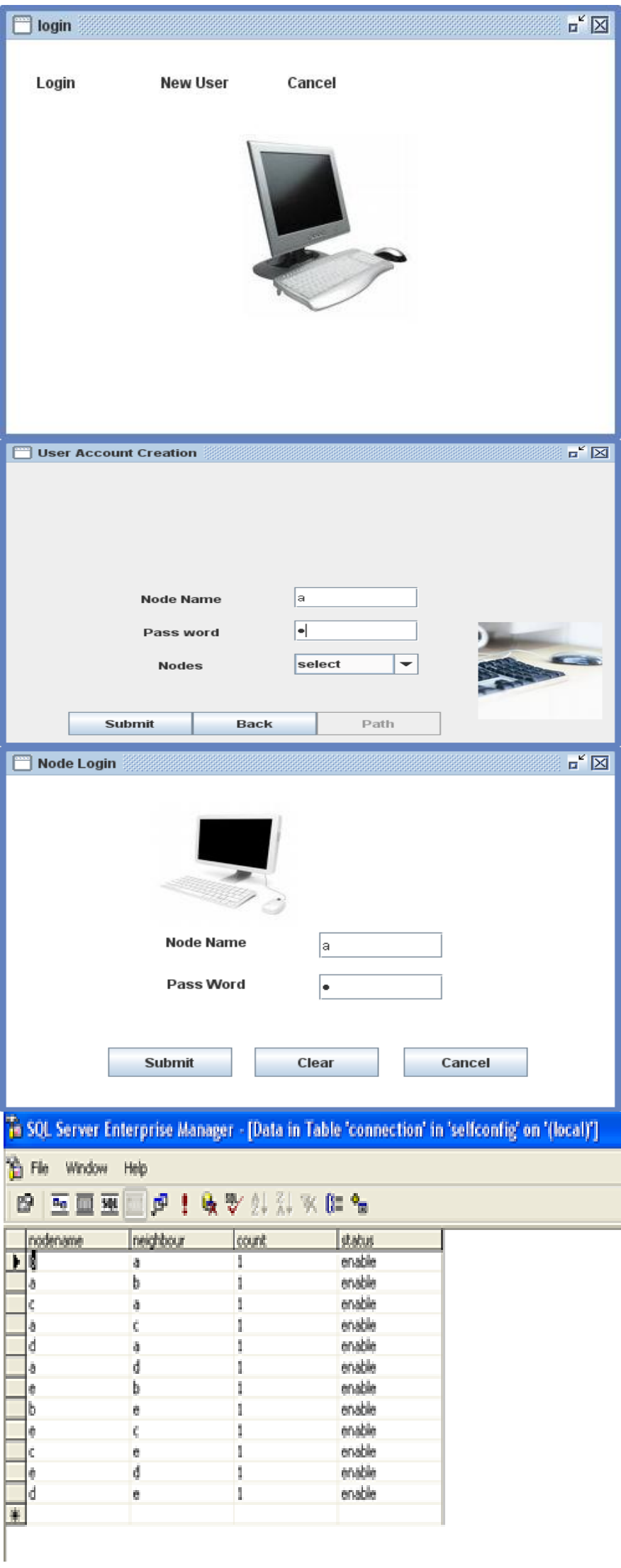

Fig.4: USER ACCOUNT CREATION AND CONNECTION DETAILS

\subsubsection{ROUTE DISCOVERY}

Send sender node request to receiver node a through all possible paths when connection established, and receive the response from receiver. We measure the available routes by getting details from server system. Calculate the path cost value for each available route. We measure the delay time for each available route from source node to destination node.We measure the throughput for each available route from source node to destination node.Fig 5 shows the possible path in the networks.

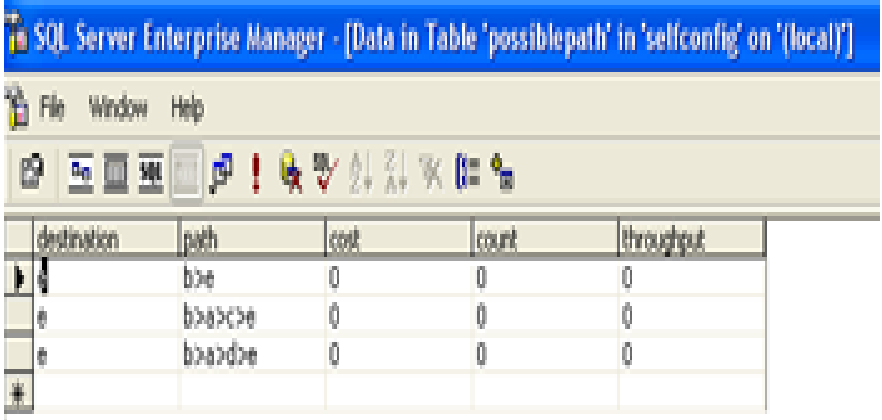

Fig. 5: POSSIBLE PATH

\subsubsection{PATH ESTIMATION}

We sort path cost values,among the path cost sort value, find out the minimum path cost, if paths are available means, process started to send data from sender node to destination node. Among that available path determine the shortest path, minimum delay time and throughput.We sends the data to receive node among these path.

\begin{tabular}{|c|c|c|c|}
\hline $9 \overline{9}$ & t & 84 & 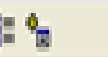 \\
\hline ath & $\omega$ & borl. & Hitotht \\
\hline 证 & 0 & 0 & 0 \\
\hline byxy & 0 & 0 & 0 \\
\hline bradse & 0 & 0 & 0 \\
\hline
\end{tabular}

\section{Fig.6: BEST PATH}

\subsubsection{FAILURE DISCOVERY}

Before sending the data, sender node will calculate threshold limit, because it is used to avoid the node failure of the sending data. While sending the data in that shortest path, It checks the threshold limit.If the threshold limit is exists, It alternatively measure the backup path that means selected path 
does not have repeated nodes which is available in previous path.Through this available path, It send the data from source node to destination node.
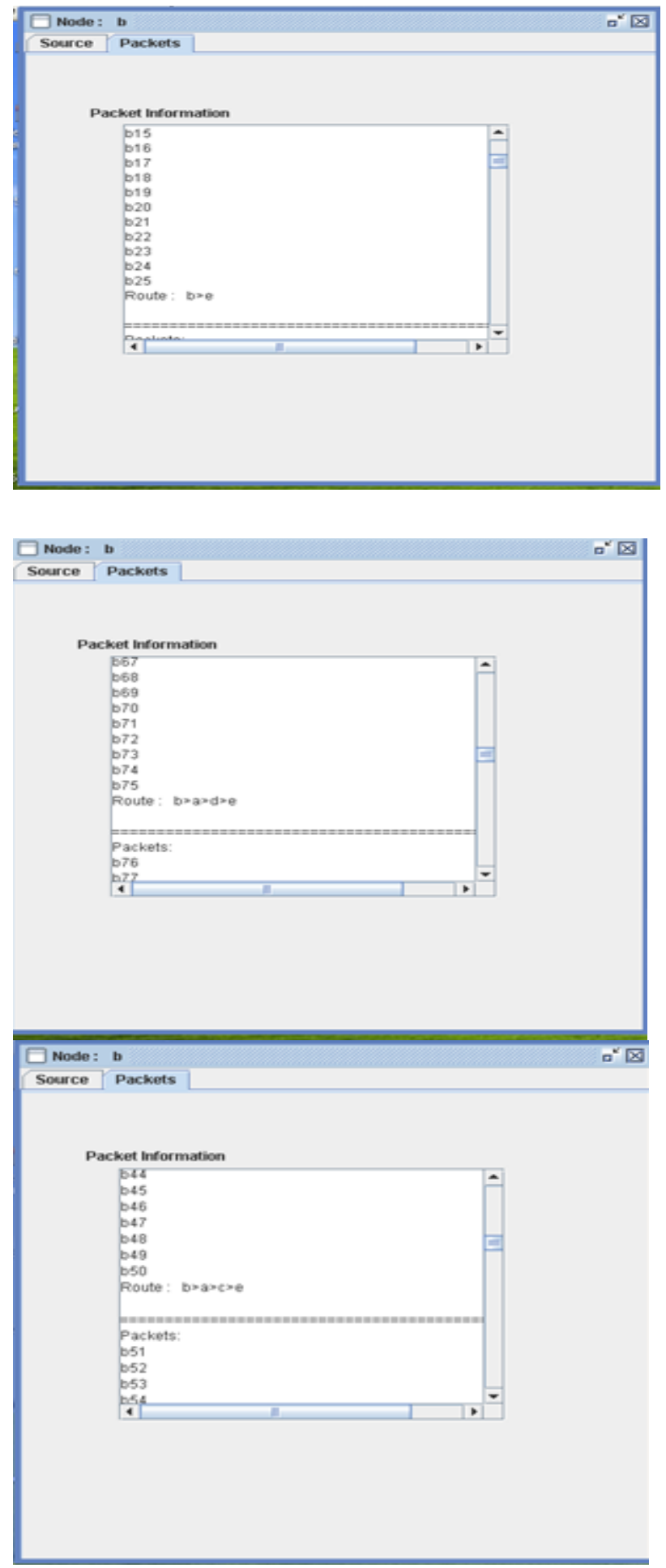
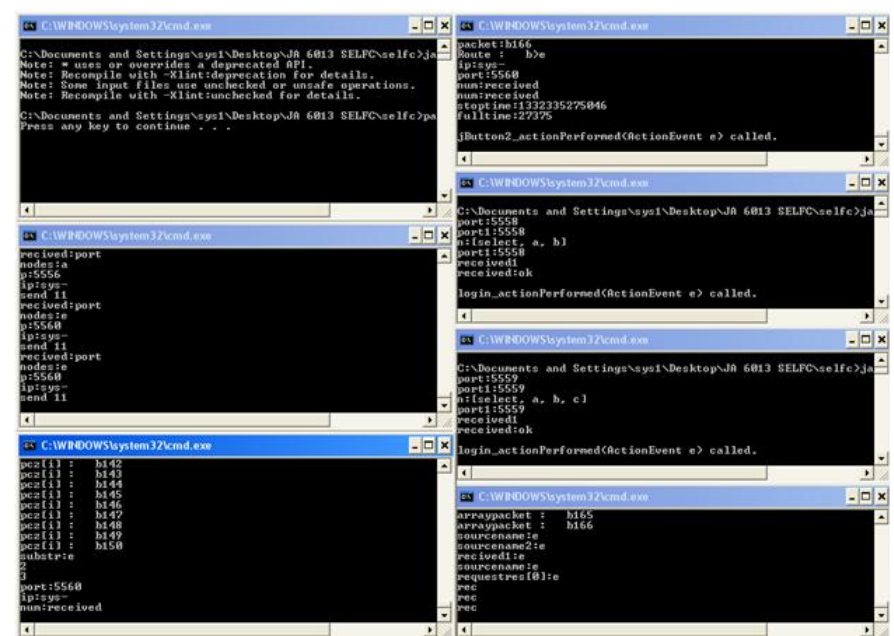

Fig. 7: PACKET ROUTING DETAILS

\subsubsection{AUTONOMOUS NETWORK RECONFIGURATION \\ SYSTEM}

ARS in every mesh node monitors the quality of its outgoing wireless links at every monitoring period. and reports the results to a gateway via a management message. Second, once it detects a link failure(s), ARS in the detector node(s) triggers the formation of a group among local mesh routers that use a faulty channel,and one of the group members is elected as a leader using the well-known bully algorithm for coordinating the reconfiguration. Third, the leader node sends a planningrequest message to a gateway. Then, the gateway synchronizes the planning requests, if there are multiple requests and generates a reconfiguration plan for the request. Fourth, the gateway sends a reconfiguration plan to the leader node and the group members. Finally, all nodes in the group execute the corresponding configuration changes, if any, and resolve the group. We assume that during the formation and reconfiguration,all messages are reliably delivered via a routing protocol and per-hop retransmission timer.

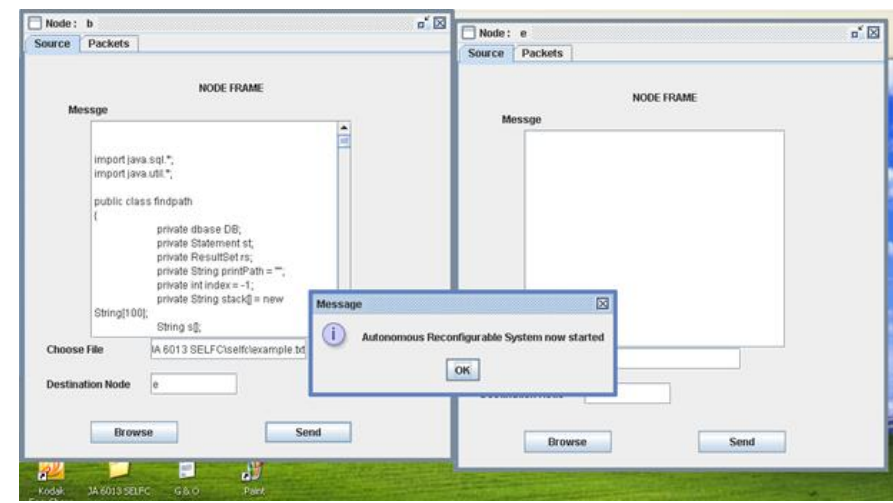




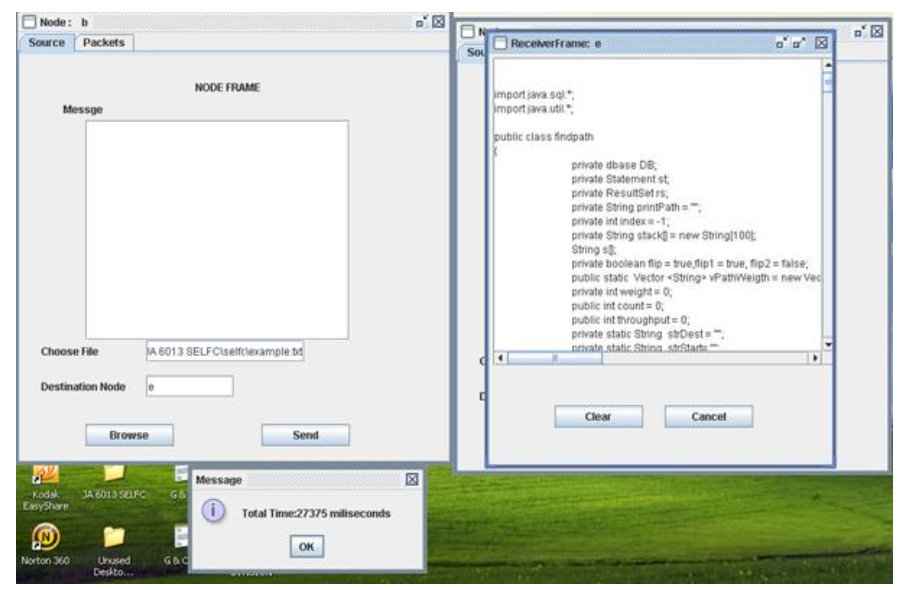

Fig. 8: DATA SENDING BY ARS

\section{EVALUATION RESULT}

Table 1 shows the comparison of the proposed system with the existing system.

\begin{tabular}{|c|l|c|c|}
\hline S.No & FEATURES & $\begin{array}{l}\text { EXISITING } \\
\text { SYSTEM }\end{array}$ & $\begin{array}{l}\text { PROPOSED } \\
\text { SYSTEM }\end{array}$ \\
\hline 1. & $\begin{array}{l}\text { PACKET } \\
\text { LOSS }\end{array}$ & YES & NO \\
\hline 2. & $\begin{array}{l}\text { THRESHOLD } \\
\text { LIMIT FOR } \\
\text { NODES }\end{array}$ & NO & YES \\
\hline 3. & $\begin{array}{l}\text { GREEDY } \\
\text { ALGORTHIM }\end{array}$ & YES & NO \\
\hline 4. & $\begin{array}{l}\text { RESOURCE } \\
\text { ALLOCATION } \\
\text { WASTAGE }\end{array}$ & YES & \\
\hline
\end{tabular}

Table 1: Evaluation result

\section{CONCLUSION AND FUTURE WORK}

\subsection{CONCLUSION}

This paper presented an autonomous network reconfiguration system (ARS) that enables a multiradioWMN to autonomously recover from wireless link failures. ARS generates an effective reconfiguration plan that requires only local network configuration changes by exploiting channel, radio, and path diversity. Furthermore, ARS effectively identifies reconfiguration plans that satisfy applications' QoS constraints, admitting up to two times more flows than static assignment, through QoSaware planning. Next, ARS's online reconfigurability allows for real-time failure detection and network reconfiguration, thus improving channel efficiency by $92 \%$. Our experimental evaluation on a Java have demonstrated the effectiveness of ARS in recovering from local link-failures and in satisfying applications' diverse QoS demands is concluded.

\subsection{FUTUREWORK}

Use of ARS in IEEE 802.11 b/g WMNs: ARS is mainly evaluated in IEEE 802.11a networks, where 13 orthogonal channels are available. However, ARS can also be effective in a network with a small number of orthogonal channels (e.g., three in IEEE $802.11 \mathrm{~b} / \mathrm{g}$ ). Because ARS includes a linkassociation primitive, it can learn available channel capacity by associating with idle interfaces of neighboring nodes, and it further limits the range of a reconfiguration group (e.g., nodes within 4 hops).

\section{REFERENCES}

[1] I Akyildiz, X. Wang and W. Wang (2005) 'Wireless mesh networks: A survey,' Comput. Netw.', Vol. 47, No. 4, pp. 445-487.

[2] P Kyasanur and N Vaidya (2005) 'Capacity of multichannel wireless networks:Impact of number of channels and interfaces', Proc. of ACM MobiCom, Cologne, Germany, pp. 43-57.

[3] K Ramanchandran, E Belding-Royer and M Buddhikot (2006) 'Interference-aware channel assignment in multi-radio wireless mesh networks', Proc. of IEEE INFOCOM, Barcelona, Spain, pp. 1-12.

[4] R Draves, J Padhye and B Zill (2004) 'Routing in multi-radio, multi-hop wireless mesh networks', Proc. of ACM MobiCom, Philadelphia, PA, pp. 114-128.

[5] M Alicherry, R Bhatia, and L Li (2005) 'Joint channel assignment and routing for throughput optimization in multi-radio wireless mesh networks', Proc.of ACM MobiCom, Cologne, Germany, pp. 58-72.

[6] M Kodialam and T Nandagopal (2005) 'Characterizing the capacity region in multi-radio multi-channel wireless mesh networks', Proc. of ACM MobiCom, Cologne, Germany, pp. 73-87. 